\title{
Mudanças dos Padrões de Morbi-Mortalidade: Uma Revisão Crítica das Abordagens Epidemiológicas
}

\author{
MAURICIO LIMA BARRETO \\ EDUARDO HAGE CARMO \\ CECI VILAR NORONHA \\ ROSANA BEZERRA BATISTA NEVES \\ PAULO CESAR ALVES*
}

Tendo seu objeto de estudo centrado nas relações do processo saúde-doença em populações humanas e sendo chamada para contribuir na reorganização das práticas sanitárias, a epidemiologia enfrenta questões teóricometodológicas cruciais. Um exemplo significativo desse tipo de questão é a utilização da análise epidemiológica para o entendimento das transformações ocorridas no processo saúde-doença dos países subdesenvolvidos. A existência de um poderoso arsenal metodológico e técnico no âmbito da epidemiologia "clássica" nem sempre atende às necessidades de novos olhares que possam desvendar outras dimensões do fenômeno epidemiológico em sociedades que apresentam intensos processos de mudanças.

* M.L. Barreto - Professor, Departamento de Medicina Preventiva e Programa de Pós-Graduaçāo em Saúde Coletiva, UFBA.

E.H. Carmo - Mestrando, Programa de Pós Graduaçāo em Saúde Coletiva, UFBA

C.V. Noronha - Socióloga, Dept Medicina Preventiva e Programa de Pós-Graduaçāo em Saúde Coletiva, UFBA.

R.B.B. Neves - Mestranido, Programa de Pós Graduaçāo em Saúde Coletiva, UFBA.

P.C. Alves - Professor, Dept de Sociologia e Programa de Pós-Graduaçāo em Saúde Coletiva, UFBA. 
Neste trabalho nos concentraremos na discussão de um aspecto controverso e relevante para o fenômeno saúde-doença: as "tendências" históricas de mudanças nos padrões de morbi-mortalidade. A bibliografia existente sobre o assunto mostra claramente que essa transformação tem ocorrido de forma intensa, e não necessariamente homogênea, em diferentes sociedades e mesmo no interior de uma dada sociedade. É possível, dentro dessa literatura, destacar dois grandes grupos de estudos. O primeiro tem buscado construir um modelo macroteórico que dê conta da globalidade do processo em diferentes países; é a "teoria da transição epidemiológica". O segundo, em uma perspectiva mais restrita, utiliza dados disponiveis com o objetivo explícito de determinar os possíveis fatores responsáveis pelas transformaçōes da morbi-mortalidade de uma dada sociedade.

Estas duas vertentes, com diferentes origens e objetivos, contribuem de maneira diferenciada para a construção do pensamento epidemiológico. Apesar da importância, ambas as perspectivas são marginalmente abordadas dentro da epidemiologia. Nosso objetivo é analisar estes dois grandes grupos, tendo em vista uma ampliação do debate teórico, tão essencial para a definição do escopo e dos limites dessa disciplina científica.

\section{Mudanças nos padrões epidemiológicos}

Todos os autores que têm trabalhado com tendências históricas dos indicadores de mortalidade, desde que os mesmos estão disponíveis nos países ocidentais, são unânimes em mostrar que há mais de um século iniciou-se um processo de constante e intenso declínio destas taxas, somente interrompido em curtos períodos, como por exemplo durante as grandes guerras. ${ }^{1}$ No tocante às tendências de morbidade, não tem sido possível desenvolver tal tipo de análise por falta de registros que permitam construir de indicadores da ocorrência de doenças em longos períodos de tempo. Entretanto, escassos dados existentes, para períodos menores, sugerem que a incidência e a prevalência de doenças também se modificam no tempo, sofrendo mudanças que nem sempre seguem em paralelo com as tendências de mortalidade. ${ }^{2}$

1. McKEOWN T., The role of medicine: dream, mirage or nemesis? Oxford, Basil Blakwell, 1979; JOSE M., "Historia universal de la mortalidad". Salud Pub. Mex., n.31, 1989, p.3-17.

2. RILEY J.C., "Long-term morbidity and mortality trends inverse health transitions". 1990, pp. 165-188. in: What we know about health transition, ed. Jonhn Caldwell, Sally Findley, Pat Caldwell, Gigi Santow, Wendy Cosford, Jennifer Braid and Daphne Broers-Freeman. Canberra:Health Transition Center, The Australian National University. 
A análise dos indicadores de mortalidade é fundamental para a compreensão da dinâmica populacional. Uma redução intensa e rápida deste indicador influencia fortemente a estrutura demográfica, com aumento na esperança de vida e envelhecimento populacional. Estas mudanças, além do mais, conduzem a transformações técnicas, financeiras e organizacionais nos serviços de saúde. Assim, cabe apresentar algumas evidências empíricas das mudanças nos padrões epidemiológicos em diferentes países para, em seguida, analisar as respostas propostas pelos teóricos.

Nos países europeus, os registros de mortalidade iniciam-se no século XVI, com os Estados se esforçando por controlar as pessoas disponíveis, tendo em vista a cobrança de impostos, a formação de exércitos etc. $\mathrm{Na}$ Inglaterra, os óbitos com especificação das causas são registrados desde 1839. ${ }^{3}$ As estatísticas deixam claro que, nos países desenvolvidos, a mortalidade começou a cair a partir da segunda metade do século XIX. Esta tendência foi acompanhada por um aumento progressivo da esperança de vida ao nascer, que passa de aproximadamente trinta anos no início do presente século para algo em torno de oitenta anos em épocas recentes. Nos Estados Unidos a esperança de vida apresentou um incremento anual de 0,2 anos entre 1880 e 1890, passando para 0,4 ano entre 1910 e 1920 . As dificuldades econômicas e a guerra interromperam esta tendência, com uma queda para 0,32/ano na década de 1930, atingindo um pico anual médio de 0,53 /ano na década seguinte. ${ }^{4}$ No Japão, que hoje tem níveis de saúde comparáveis aos outros países desenvolvidos, a mudança ocorreu de forma bastante intensa, pois a mortalidade só caiu acentuadamente a partir da segunda década deste século. ${ }^{5}$

As mudanças registradas nas taxas gerais de mortalidade são mais bem entendidas quando decompostas por grupos de causas. Nesses países, verifica-se uma substituição das doenças infecciosas e parasitárias (DIP) pelas doenças crônico-degenerativas (DCD) e por causas externas como principais componentes da mortalidade, em termos proporcionais. $\mathrm{Na}$ Inglaterra, no período de 1848 a $1971,74 \%$ de redução nas taxas de mortalidade foram atribuídos ao declínio das DIPs. ${ }^{6}$

A partir de 1968 foram observadas nos Estados Unidos taxas declinantes no grupo das $\mathrm{DCD}$, às custas principalmente das doenças cardiovascu-

3. ALDERSON M., An introduction to epidemiology. 2a.ed., London, The Macmillan Press Ltd, 1983.

4. GWATKIN D.R., "In dications of change in developing country mortality trends: the end of an era?", Pop. Dev. Rev., n.6, 1980, p. 615-44.

5. OMRAN A.R., "The epidemiologic transition: a theory of the epidemiology of population change", Milbank Mem.Fund.Q., n.49, 1971, p. 509-83.

6. McKEOWN T., op. cit. 
lares (DCV). Pouco tempo depois, o mesmo fenômeno teve início em outros países desenvolvidos, como Holanda, Japão, Canadá e Bélgica. Para se ter uma idéia do significado desta redução, as taxas de mortalidade por DCV ajustadas por idade, caíram nos Estados Unidos, no período de 1968 a 1987, em aproximadamente $40 \%$. Entretanto, mesmo em alguns países desenvolvidos, como Alemanha, Áustria, França e Dinamarca, estas taxas continuaram aumentando.?

Ora, se a mortalidade passa a decorrer principalmente de patologias crônicas, o prolongamento da vida coloca novos problemas com relação à morbidade. Esta, para muitos grupos de causa, tem apresentado tendência crescente, assumindo portanto um sentido inverso ao padrão de mortalidade. Isso traz consequiências sobre a qualidade da vida, por aumentar o tempo médio das incapacidades. ${ }^{8} \mathrm{~A}$ melhoria das condições de vida passa, assim, por aquilo que Fries ${ }^{9}$ denominou de "compressão da morbidade", caracterizada por um encurtamento do período compreendido entre a ocorrência de doenças/incapacidades e a idade correspondente à expectativa de vida.

Nos países ditos subdesenvolvidos - um grupo bastante heterogêneo sob diversos aspectos - a tendência declinante da mortalidade só é observada de forma mais consistente e generalizada a partir da década de 1940. Entretanto, declínios significativos em períodos mais remotos têm sido observados, como por exemplo na América Latina no início do século. ${ }^{10}$ Deve-se observar que nestes países, em geral, os registros de mortalidade são deficientes, o que dificulta a construção de séries históricas das taxas de mortalidade para períodos longos. Este problema é mais grave nos diversos países do continente africano.

Com raras exceções, o ponto em comum entre os países subdesenvolvidos é que, independentemente das tendências observadas, apresentam diferenças acentuadas quando comparados aos desenvolvidos. Naqueles

7. PISA Z. e UEMURA K. "Trends of mortality from ischaemic heart disease and other cardivascular diseases in 27 countries, 1986-1977", Wld. Hlth. Stat. Quart., n.35, 1982, p.11-48; UEMURA K. e PISA Z. "Recent trends in cardiovascular disease mortality in 27 industrialized countries." Wld. Hlth. Stat. Quart., n.38, 1985, p.142-9.

8. VERBRUGGE L.M., "Longer life but worsening health? Trends in health and mortality of middle-aged and older persons", Health and Society, v.62,n.3,1984. p.475-519; RILEY J.C., op.cit.; COLVEZ A. \& BLANCHET M., "Disability trends in the United States population 1966-76: analysis of reported causes", AJPH, v.71, n5, 1981, p.464-70.

9. FRIES J.F., Aging, natural death, and compression of compression of morbidity. N.Eng.J.Med.,v.303,n.3,1980, p.130-135; Society, v.61, n.3, 1983, p.397-419.

10. ARRIAGA E.E. e DAVIS K., "The pattern of mortality change in Latin America", Demography, v.6, n.3, 1969, p.223-242. 
países, as taxas de mortalidade apresentam-se altas, com alto peso relativo das DIPs, e a expectativa de vida ao nascer apresenta diferença média da ordem de vinte anos com a dos países desenvolvidos. ${ }^{11}$ Entretanto, em muitos países, particularmente na Europa Oriental, Ásia e América Latina, as doenças crônico-degenerativas já são responsáveis pela maior proporção dos óbitos.

Merece especial atenção, nesse grupo de países, os integrantes do antigo bloco socialista. Em países como China, Cuba e Hungria observaram-se, após suas respectivas revoluções, rápida queda nas taxas de mortalidade e na preponderância das DIPs, bem como aumentos substanciais na expectativa de vida. Porém, este avanço apresenta percalços. $\mathrm{Na}$ Hungria, seguindo-se a estas tendências observadas a partir do a pós-guerra até meados da década de 1960 , verificaram-se, até o final da década de 1980, elevações das taxas de mortalidade e queda, ainda que discreta, na expectativa de vida ao nascer. ${ }^{12}$

Observando-se em maiores detalhes esse processo na América Latina, verifica-se que as modificações das tendências de mortalidade têm-se caracterizado não só por seu início tardio, mas também pela grande heterogeneidade entre os diversos países da região.

De uma maneira geral, houve um ganho na expectativa de vida ao nascer para toda a região, elevando-se de quarenta anos em 1930 para 64 anos em 1980. Entretanto, esse processo se dá de forma desigual. Assim, no que diz respeito ao coeficiente de mortalidade infantil (CMI), observou-se que na década de 1980 Cuba e Costa Rica apresentaram CMI de 20 por 1000 nascidos vivos, enquanto Haiti e Bolívia apresentaram um CMI seis vezes superior. Na composição da mortalidade por grupo de causas, no mesmo período, observou-se que as DCVs eram responsáveis por 5\% dos óbitos na Guatemala, El Salvador e Equador, enquanto em Cuba, Costa Rica, Chile e Argentina este grupo de causas representava, em média, 30\% dos óbitos. ${ }^{13}$

No Brasil, a partir da década de 1960 , seguindo-se a um intenso processo de crescimento populacional observado desde o início do século, configurase uma desaceleração dessa tendência, em função de uma queda da taxa de

11. GWATKIN D.R., op.cit.

12. RUZICKA L. e KANE P., "Health transition: the course of morbidity and mortality", 1990,p.1-26. in: What we know about health transition, op. cit.; JÓZAN P., "Some features of mortality in postwar Hungary: the third epidemiological transition", Cah. Socio. Demo. Med, XIXème annee. n.1, 1989, p.21-42.

13. FRENK J., FREJKA T., BOBADILLA J.L., STERN C., LOZANO R. e JOSE M., 'La transición epidemiologica en America Latina", Bol. of Sanit. Panam.,v.111, n.6, 1991, p. 485-96. 
natalidade, mais acentuada do que a verificada nas taxas de mortalidade. ${ }^{14}$ A redução nas taxas de natalidade persistiu nas décadas subseqüentes e tem ocorrido paralelamente a uma queda da mortalidade infantil, que no período de 1941 a 1984 apresentou uma redução de $63,4 \%$, em que pese as flutuações anuais observadas. ${ }^{15}$ Como conseqüência verifica-se um aumento significativo na expectativa de vida ao nascer, que passa de 33,7 anos, no início do século, para 65,5 anos em $1980,{ }^{16}$ iniciando-se assim o processo de envelhecimento da população brasileira. Ainda que o percentual de idosos no Brasil (6\%), fosse inferior em 1980 à média verificada nos países europeus (17\%), projeções para o início do próximo milênio indicam que este grupo representará segmento importante, o que influenciará o padrão epidemiológico e as demandas dos serviços de saúde.

As modificações na composição da mortalidade por grupo de causa se tornam mais nítidas no Brasil a partir de 1930. Naquele ano, as DIPs representavam $45,7 \%$ do total de óbitos, caindo para $8 \%$ em 1986. Os óbitos por DCV representavam, em 1986, 33,5\% do total, seguido do grupo de causas externas, responsáveis por $14,9 \%$ dos óbitos. ${ }^{17}$ Este último grupo de causa vem apresentando tendência crescente, destacando-se os homicídios, com taxas muito elevadas, superiores às dos Estados Unidos, Canadá e alguns países europeus. As neoplasias também vêm aumentando sua participação na composição da mortalidade, sendo que o maior incremento deve-se aos cânceres de pulmão e de mama, enquanto outras neoplasias, tais como câncer de cervix uterino, têm diminuído sua participação. ${ }^{18}$

As tendências de mortalidade para o país apresentam grandes diversidades regionais, tendo-se como exemplo as DIPs, que em 1986 constituíam a terceira causa de óbito na Região Nordeste e a sexta causa nas regiões Sul e Sudeste. ${ }^{19}$ Em 1980, a Região Nordeste apresentava uma esperança de vida ao nascer de 51 anos, enquanto a Região Sul atingia 67,2 anos. ${ }^{20}$

14. BAYER G.F., ROTBERG L.C., TAVARES R. e PAULA S.G., "Populaçāo brasileira no século XX: alguns dados", RADIS/FIOCRUZ, Dados. n.2, 1982. p.1-8.

15. YUNES J. e RONCHEZEL V.S.C., "Evolução na mortalidade geral, in fantil e proporcional no Brasil", Rev. Saude Publ., n. 8 (suppl.), 1974, p.3-48; RAMOS R.R., VERAS R.P. e KALACHE A., "Envelhecimento populacional: uma realidade brasileira", Rev. Saude Publ., v.21, n.3, 1987, p.211-24.

16. KALACHE A., VERAS R.P. e RAMOS L.R., "O envelhecimento da população mundial. Um desafio novo", Rev. Saude Publ., v.21, n.3, 1987, p.200-10.

17. BAYER G.F. e PAULA S.G., "Mortalidade nas capitais brasileiras 1930-1980", RADIS/FIOCRUZ, Dados. n.7, 1984. p.1-8; MINISTÉRIO DA SAÚDE. Fundaçāo Nacional de Saúde.Subsistema de Informaçōes sobre Mortalidade. Estatísticas de mortalidade - Brasil:1986. Brasília, Centro de Documentação do Ministério da Saúde, 1991.

18. WORLD BANK, Brazil: the new challenge of adult health. Washington DC, World Bank,1990.

19. MINISTÉRIO DA SAÚDE. Fundaçāo Nacional de Saúde. Subsistema de Informaçōes sobre Mortalidade, op.cit. 


\section{Percalços na construção de uma teoria: a transição epidemiológica}

Uma das mais importantes perspectivas para a interpretação das mudanças nos padrões de morbi-mortalidade tem sido a teoria da transição epidemiológica. Ela foi originalmente desenvolvida para dar suporte à teoria da transição demográfica, ${ }^{21}$ na medida em que os eventos mórbidos têm fortes implicações na dinâmica populacional. As teorias demográficas de base neo-malthusiana explicam as experiências exitosas de desenvolvimento econômico como conseqüência da redução da mortalidade e da fertilidade. A redução da mortalidade seria resultante da assimilação de novas tecnologias que aumentam a efetividade e a eficiência dos serviços de saúde, independentemente dos níveis de produção e consumo. ${ }^{22}$ Os mais radicais defensores daquela teoria acreditam que medidas de saúde pública, com alto impacto na mortalidade, não devem ser utilizadas em áreas subdesenvolvidas, nas quais são altas as taxas de crescimento populacional.

Frederiksen ${ }^{23}$ propõe um modelo alternativo, que se fundamenta na evidência de que as diminuições da mortalidade e da fertilidade ocorrem como conseqüência da elevação do nível de vida. A queda nas taxas de mortalidade é vista, por este autor, como parte da solução dos problemas populacionais, conjuntamente com a redução da fertilidade, e não como determinante do desenvolvimento econômico. A redução da mortalidade passa a ser vista como um dos fatores contributórios para a redução da fertilidade. Este autor é o primeiro a estabelecer a idéia de que os padrões de doença e reprodução ocorreriam em estágios (tradicional, transicional precoce, transicional tardio e moderno), refletindo o grau de modernização da sociedade. Para cada um desses estágios corresponderiam padrões definidos de problemas ambientais, doenças predominantes, mortalidade, fertilidade, estado nutricional e organização dos serviços de saúde.

Posteriormente Omram,${ }^{2 \overline{4}}$ sem fazer referência ao trabalho de Frederiksen, tomou como base a teoria da transição demográfica para estabelecer o conceito de transição epidemiológica. Este constitui um processo universal, fundamentado nas seguintes proposições: a) a mortalidade é um

20. IBGE, Indicadores sociais, tabelas selecionadas, v.2, 1984.

21. AN NORTH L. \& KARON B.P., "Demographic transition re-examined", Am.Sociol.Rev., n.20, 1955, p.523-7.

22. CIPOLLA C.M., The economic history of world population. Penguim, Baltimore, 1964.

23. FREDERIKSEN H., "Feedbacks in economic and demographic transition", Science., n.166, 1969, p.837-47.

24. OMRAN A.R., op.cit. 
importante fator na dinâmica populacional; b) as pandemias são gradualmente substituídas por doenças degenerativas e outras doenças conseqüentes à ação do homem; c) durante a transição epidemiológica, as mudanças mais profundas ocorrem entre crianças e mulheres jovens; d) as mudanças que ocorrem nos padrões de saúde e doença acompanham a transição demográfica e socioeconômica, fazendo parte do complexo de modernização. Para o autor, o processo transicional ocorreria em três estágios: a idade das pestilências e da fome, a idade do declínio das pandemias e a idade das doenças degenerativas aliadas a outras doenças criadas pelo homem. Olshansky \& Ault, ${ }^{25}$ a partir da observação da queda da mortalidade por algumas doenças crônicas, definiram posteriormente um quarto estágio, a idade do prolongamento das doenças degenerativas. Com base em observações de situações concretas, Omran ${ }^{26}$ define que os estágios poderiam ocorrer em três padrões: a) o modelo clássico, ou ocidental, representado pela experiência dos países da Europa Ocidental e da América do Norte; b) o modelo acelerado, cujo exemplo mais expressivo é o do Japão, caracterizado por uma queda acentuada das suas taxas de mortalidade a partir da segunda década deste século; e c) o modelo tardio, ou contemporâneo, que descreve mudanças recentes, ainda em processo nos países subdesenvolvidos.

A idéia de transição epidemiológica, como visto, está associada com a busca de uma estrutura macroteórica capaz de ordenar a ampla variedade de observações comparativas sobre as mudanças nos padrões de mortalidade e morbidade entre as nações. Seus fundamentos são decorrentes do enfoque neo-evolucionário da modernização. Trata-se, nesse aspecto, de um conceito baseado na suposição de que as sociedades "tradicionais" (em grande parte, rurais) se transformam em sociedades "adiantadas" ou "modernas" mediante uma seqüência determinada de etapas específicas. ${ }^{27}$ Assim, o modo de viver tradicional/primitivo seria gradualmente substituído por um modo de viver moderno/dinâmico. O subdesenvolvimento e o desenvolvimento seriam os extremos opostos de um processo contínuo. A modernização é apresentada como uma transformação total da sociedade tradicional (pré-moderna) nos tipos de tecnologia, urbanização e organização social que caracterizam as nações adiantadas e economicamente prósperas. ${ }^{28}$ Conseqüentemente, o conceito de transição epidemiológica assume uma dimensão teleológica.

25. OLSHANSKY S.J. \& AULT A.B., "The fourth stage of the epidemiologic transition: the age of delayed degen erative diseases", Milbank Mem. Fund. Q. v.64, n.3, 1986, p.355-91.

26. OMRAN A.R., op.cit.

27. ROSTOW W.W., Etapas do desenvolvimento econômico. 5a.ed., Rio de Janeiro, Zahar Editores, 1974. 
Existem vários modelos teóricos que procuram explicar esses processos de transformação. A influência da teoria do desenvolvimento social proposta por Parsons, contudo, é dominante em todos esses modelos. ${ }^{29} \mathrm{O}$ elemento básico de explicação está no pressuposto de que existem diferenciações estruturais marcantes entre as sociedades "modernas" e as "tradicionais". Essas diferenças, descritas em termos de uma ordem de complexidade morfológica, tendem a ser mutuamente exclusivas e conflitantes. A coexistência das duas estruturas em uma dada nação, conforme esse raciocínio, evidencia um estado de "transição" de uma sociedade tradicional para uma sociedade moderna.

Os modelos explicativos da transição epidemiológica propostos por Omran, ${ }^{30}$ Frederiksen ${ }^{31}$ e Olshansky e Ault ${ }^{32}$ tendem a adotar uma visão simples, evolucionista e etnocêntrica da mudança: a idéia de um padrão de desenvolvimento linear e unidirecional, calcada naquilo que parece ter acontecido em países capitalistas da Europa Ocidental e nos Estados Unidos. Esse pressuposto parece insustentável. Em prímeiro lugar, esse modelo explicativo postula a existência de processos estruturais semelhantes e extrapola (universaliza) um ponto de vista sobre as seqüências históricas ocorridas naqueles países. Ao retratar as regularidades estruturais gerais no processo de mudança, tais análises concentram atenção em duas ou três variáveis e isolam muitas outras. Esse tipo de enfoque é metodologicamente problemático porque, ao enfatizar essas variáveis, o analista tende a subestimar ou tomar como constante a complexidade estrutural dos fatores sociais. Por não levar em sua devida consideração a heterogeneidade do conjunto de países, esse modelo fica restrito a uma concepção: a) embrionária e excessivamente abstrata da mudança social e b) de cadeias lineares de determinação (causação unidirecional). Dessa forma, a procura de uma homogeneização não provoca maior esforço de teorização.

A abordagem da modernização, em segundo lugar, não leva em devida consideração que o modelo ocidental de desenvolvimento foi resultante de uma revolução industrial vinda de dentro, enquanto os países contemporâneos, subdesenvolvidos, experimentam uma "revolução" vinda de fora, trazida pelo impacto da tecnologia ocidental. Em uma visão etnocêntrica e teleológica do desenvolvimento, os teóricos da modernização esperam

28. GERMANI G., Sociologia da modernização. Sāo Paulo, Ed. Mestre Jou, 1974.

29. WEBSTER A., Introduction to the sociology of development. Hampshire, Macmillan, 1984.

30. OMRAN A.R., op.cit.

31. FREDERIKSEN H., op.cit.

32. OLSHANSKY S.J. \& AULT A.B., op.cit. 
encontrar nos países subdesenvolvidos exemplos semelhantes aos dos países europeus.

Coerente com a visão idealizada do desenvolvimento europeu, o modelo permite supor uma coexistência do sistema tradicional e moderno em uma mesma nação. Essa visão dual, ou dualista, retrata a estrutura subdesenvolvida como dividida em dois setores contrastantes e, em grande parte, independentes: o rural/tradicional e economicamente atrasado, e o setor urbano/moderno, industrializado, que proporciona a dinâmica para a mudança. ${ }^{33}$

Em terceiro lugar, boa parte dos modelos explicativos da transição epidemiológica repousa em uma perspectiva eminentemente estrutural, para explicar a mudança nos padrões de morbi-mortalidade. Trata-se de uma perspectiva que procura formular proposições altamente generalizadas e deterministas sobre os tipos de mudança estrutural que devem ocorrer, sob o impacto de determinados fatores. Não pode, porém, tratar de forma adequada a variabilidade e flexibilidade dos sistemas sociais, a questão das reações diferenciais à mudança, evidenciada pelos diferentes grupos e categorias sociais de uma dada população. Ao focalizar sua análise no aspecto macroteórico da sociedade, a perspectiva estrutural perde de vista o processo pelo qual os indivíduos e os grupos respondem às mudanças de seu ambiente. Além disso, os estudos da transição epidemiológica não têm dado a devida ênfase no exame da intervenção de políticas estatais, intimamente relacionadas com a promoção de certos tipos de mudança estrutural e orgânica. As mudanças na política do governo podem levar ao desenvolvimento de novos padrões de organização ou, alternativamente, ao fortalecimento de estruturas "tradicionais".

O enfoque neo-evolucionário da modernização tem sido amplamente contestado por diversos teóricos do desenvolvimento social. ${ }^{34}$ Nos estudos mais recentes da transição epidemiológica, os modelos desenvolvidos por Lerner ${ }^{35} \mathrm{e}$, posteriormente, por Frenk et. al. ${ }^{36}$ contestam, em alguns aspec-

33. REDFIELD R., "The folk society", Am.J.Sociol., n.52, 1947, p.292-308.

34. WIARDA M.J., "Por uma teoria não-etnocêntrica do desenvolvimento: as concepçōes alternativas do $3^{\circ}$ mundo",.Dados, vol.25, n.2, 1982, p. 229-51; SOUZA G.A.A. de A., "A hipótese da convergência: uma ilusāo de ótica", Caderno CRASS, n.15, 1991, p.47-69; CARDOSO F.H., "As idéias e seu lugar: ensaios sobre as teorias do desenvolvimento", Petrópolis, Vozes, 1980.

35. LERNER M., Modernization and health: a model of the health transition. Trabalho apresentado na Reuniāo Anual da American Public Health Association, San Francisco, California, 1973.

36. FRENK J., FREJKA T., BOBADILLA J.L., STERN C., LOZANO R. e JOSE M., op.cit.; FRENK J., BOBADILLA J.L., SEPULVEDA J. e CERVANTES M.L., "Health transition in middle-income countries: new challenges for health care", Health Policy Planning, v.4, n.1, 1989, p.29-39. 
tos, as premissas básicas de um sistema global unificado de mudança, tal como proposto por Omran, Frederiksen, Olshansky e Ault.

Ao examinar as condições de saúde na América Latina, Frenk et.al. ${ }^{37}$ distingue o estudo das mudanças nos processos de saúde e enfermidade, que definem o perfil epidemiológico de uma população (o conceito de transição epidemiológica em sentido restrito) e a resposta socialmente organizada a tais mudanças (transição da atenção sanitária). A premissa básica desses autores é de que os países latino-americanos são qualitativamente diferentes das nações desenvolvidas e reproduzem formas socioestruturais diferenciadas. Observam que a perspectiva linear e unidirecional subjacente à noção de transição epidemiológica não se aplica a esses países. As diversidades nos padrões de mortalidade e enfermidade nesse continente nos conduzem, conforme esses autores, a um novo modelo de transição epidemiológica. Pela análise dos seus padrões de transição, os países latino-americanos podem ser classificados em três grupos básicos: a) aqueles que se encontram em uma etapa avançada de transição, dentro de uma modalidade um tanto semelhante à dos países desenvolvidos (Cuba, Costa Rica e Chile, por exemplo); b) os países que se encontram em uma etapa inicial de transição (Haiti, Bolívia e Peru); c) e os países, como México e Brasil, que chegaram a uma etapa avançada mas que apresentam um modelo diferente de transição. Para esse terceiro grupo é proposto o nome de "modelo polarizado prolongado". Esse modelo tem como características: a) superposição de etapas: as doenças infecciosas superpõem-se às não infecciosas e ambas são causas importantes de morbi-mortalidade, tan to absoluta como relativa; b) contratransição: doenças erradicadas ou em estágios avançados de controle ressurgem e se transformam em importantes problemas de saúde, como a malária e o cólera; c) transição prolongada: permanece uma situação de morbi-mortalidade mista em que se mantém uma grande incidência de doenças infecciosas, sem que se chegue ao predomínio absoluto dos processos crônicos; d) polarização epidemiológica: as três características antes descritas afetam desigualmente diferentes grupos sociais, fazendo com que a heterogeneidade seja um fator marcante, tanto entre países como dentro deles próprios, o que explicaria, inclusive, a natureza prolongada da transição.

O modelo explicativo de Frenk está, até certo ponto, associado com a "teoria da articulação". Essa teoria corresponde a uma resposta aos desafios provocados pela teoria da modernização. O conceito de "articulação", formulado inicialmente por Claude Meillassoux e Pierre Philippe Rey,

37. FRENK J., FREJKA T., BOBADILLA J.L., STERN C., LOZANO R. e JOSE M., op.cit. 
significa a manifestação concreta da coexistência hierárquica de diferentes modos de produçāo dentro de uma dada formação social. Parte-se, portanto, de uma diferença entre o conceito abstrato de modo de produção (por exemplo, feudalismo, capitalismo) e de formação social (que pode conter diferentes modos de produção, pois a transição de um modo de produção para outro nunca é súbita ou completa). Assim, o conceito de articulação refere-se a uma coexistência de diferentes estruturas socioeconômicas em uma mesma sociedade.

Cabe observar que a análise de Frenk et. al. sobre a transição epidemiológica na América Latina ${ }^{38}$ é menos passível de sofrer as acusações feitas aos modelos construídos sob o enfoque neo-evolucionário da modernizaçāo. Esses autores apresentam uma visão mais flexível. Trata-se de um estudo que leva em conta as variações nas características estruturais de diferentes tipos de sociedades. Mas a tentativa de identificar os vários modelos de transição ainda dá a impressão de que também esses autores estão argumentando do ponto de vista de um modelo ocidental de desenvolvimento. Assim, por maiores que sejam as diferenças entre a abordagem de Frenk e a dos teóricos da modernização, ambos formulam um modelo linear, generalizado ou centralizado, do desenvolvimento, ao atribuir, em suas análises, prioridade ao papel dos fatores exógenos na promoção da mudança e na determinação das estruturas locais e regionais. Não dão suficiente atenção às maneiras pelas quais os grupos e processos locais podem contribuir para modificar os padrões de desenvolvimento regional e nacional.

\section{Os estudos empíricos e a interpretação das mudanças das taxas de mortalidade}

Enquanto a teoria da transiçāo epidemiológica caracteriza-se pela busca de estruturas macroteóricas que possam sistematizar as mudanças nos padrões de morbi-mortalidade entre diferentes nações, os estudos empíricos - inadequadamente denominados de "ecológicos" na epidemiologia - procuram, para sociedades isoladas, possíveis determinantes dessas mudanças. Um dos marcos significativos neste campo é o trabalho de McKeown, ${ }^{39}$ que analisou as tendências das taxas gerais e específicas de mortalidade na Inglaterra desde 1848 e concluiu que as mudanças registradas deveram-se, em grande parte, à redução das taxas de mortalidade

38. FRENK J., FREJKA T., BOBADILLA J.L., STERN C., LOZANO R. e JOSE M., op.cit.

39. McKEOWN T., op. cit. 
por doenças infecciosas. Tal fato decorreu, em primeiro lugar, da melhoria do estado nutricional da população, seguida da diminuição da exposição aos agentes infecciosos como conseqüência das medidas de saneamento ambiental - implementadas, sobretudo, em meados do século XIX - e da redução do crescimento populacional, determinada pela mudança no padrão reprodutivo. Nesse aspecto, Mckeown minimiza o papel das tecnologias médicas curativas (principalmente antibióticos) ou preventivas (principalmente vacinas e quimioprofiláticos), introduzidas com maior intensidade a partir da década de 1940, quando grande parte do declínio já teria ocorrido. Considera a Revolução Industrial e as reformas sociais do século XIX fatores que geraram as condições para uma maior produção de alimentos, uma melhoria nas condições de saneamento, mudanças culturais e o desenvolvimento da tecnologia médica.

Seus achados foram corroborados por McKinlay \& McKinlay. ${ }^{40}$ Analisando as tendências de mortalidade nos Estados Unidos desde 1900, eles concluem que o impacto das tecnologias sobre as doenças infecciosas explicam, no máximo, $3,5 \%$ da ređução da mortalidade no país.

Ainda para a Inglaterra, José ${ }^{41}$ assinala que a redução da mortalidade por tuberculose, geralmente atribuida à terapêutica antimicrobiana, era uma tendência que se evidenciava desde 1860, quase um século antes da introdução desta medida. Da mesma forma, a metade da redução na mortalidade por difteria foi obtida antes do aparecimento de qualquer medida tecnológica específica. $O$ autor atribui tais tendências nos padrões de mortalidade à melhoria das condições de vida e do meio ambiente, bem como do padrão alimentar.

Da mesma forma, tem sido relativizado o papel das tecnologias médicas no atual declínio da mortalidade devida a doenças crônicas em países desenvolvidos, enfatizando-se o papel da prevenção, que decorre de mudanças nas condições de vida e nos hábitos da população. ${ }^{42}$

Estes posicionamentos são extremamente importantes na medida em que se contrapõem aos defensores da denominada "hipótese médica", defendida arduamente pelo establishment médico com o beneplácito e o

40. McKINLAY J.B. e McKINLAY S.M., "The questionable contribution of medical measures to decline of mortality in the United States in the twentieth century". Milbank Mem. Fund. Q., n.55, 1977, p.405-28.

41. JOSÉ M., op.cit.

42. McKINLAY J.B., McKINLAY S.M. e BEAGLEHOLE R., "A review of the evidence concerning the impact of medical measures on recent mortality and morbidity in the United States", Int.J.HealthServ., v.19, n.2,1989, p.181-208. 
interesse das grandes corporações industriais farmacêuticas e de equipamentos para o setor saúde. ${ }^{43}$

Ainda que alguns trabalhos se alinhem aos questionamentos do papel da tecnologia médica nas modificações dos padrōes de mortalidade, outros trazem conclusões, algumas vezes, opostas. Nesta linha, Doll, ${ }^{44}$ verificando a redução da mortalidade por neoplasias e doença isquêmica do coração na Inglaterra e no País de Gales, atribui esta tendência à melhoria nos métodos diagnósticos, terapêuticos e, em menor escala, às modificações de hábitos em grupos populacionais de risco.

A controvérsia assinalada está centrada numa atitude subjetiva frente à in terpretação de um mesmo processo, podendo ser o reflexo de uma falsa dicotomia entre utilização de tecnologia e desenvolvimento econômico. $O$ efeito da utilização de determinadas tecnologias médicas (ou de procedimentos médicos) sobre a mortalidade de uma população tem sido observado na melhoria de alguns parâmetros relativos às suas condiçōes de vida, como foi verificado para a reduçāo da mortalidade infantil na América Latina no período de 1900 a $1985 . .^{45}$ Nesse estudo, foi observado que essa redução estava associada às intervenções governamentais em uma escala social mais ampla (abastecimento de água, saneamento), bem como às melhorias no estado nutricional dos recém-nascidos e nos cuidados pré e pós-natais. Questiona-se, nesse trabalho, o papel da terapêutica antimicrobiana e da vacinação na redução da mortalidade infantil durante o período do pós-guerra, pois a utilização destas tecnologias se deu em meados deste século, quando, para muitos países, a redução já vinha sendo observada.

Estudos empíricos têm demonstrado que o efeito das ações médicas e técnicas de saúde pública sobre a mortalidade infantil é limitado, quando são mantidas as precárias condições de vida da população. ${ }^{46}$ Corroborando esta afirmação, Paim, ${ }^{47}$ investigando as possíveis causas da elevação do coeficiente de mortalidade infantil em Salvador (Bahia), que ocorreu no período de 1968 a 1973, observou que, nesse período, houve um aumento do número de consultas pediátricas pela rede previdenciária. Isto o levou

43. WING S., "The role of medicine in the decline of hypertension-related mortality", Int.J.Health Serv., v.14, n.4, 1984, p.649-67.

44. DOLL, R., "Demographic and epidemiologic trends today Arzneim", -Forsch./Drug Res., v.39, n.II, 1989, p.943-6.

45. PALLONI, A., "Health levels and care in Latin America: the case of infant mortality 1900-1985", 1990, p.189-212. in: What we know about health transition op. cit..

46. BEHM H.; MARCHANT L.; MARIN M.; MUSSIET D.; ORTIZ S. \& TAUCHER E., "Mortalidad infantil en Chile: tendencias recientes", Cuad.Med.-Soc., v.11, n.3., 1970, p.5-13.

47. PAIM J.S., Variáveis ambientais e mortalidade infantil no municipio de Salvador. Tese apresentada à Faculdade de Medicina da Universidade Federal da Bahia, 1975. 
a concluir que a redução real do salário mínimo, observada pelo autor, foi um fator limitante para a persistência do declínio daquele coeficiente, que vinha se dando a partir de 1962, não tendo a assistência médica conseguido evitar a sua posterior elevação.

Outro ponto importante é que as medidas médicas demostram efetividade, com diferentes intensidades, sobre grupos populacionais específicos. Em estudo realizado na Inglaterra, Mackenbach et. al. ${ }^{48}$ acentuam que, entre 1931 e 1961 - e, menos consistentemente, no período subseqüente até 1981 - a redução da mortalidade por "doenças vulneráveis" às medidas médicas foi verificada em maior proporção para classes ocupacionais superiores, fenômeno que pode ser parcialmente atribuído às desigualdades no acesso, utilização e qualidade da assistência médica.

Apesar da importância desses estudos empíricos no debate contemporâneo sobre os determinantes do processo saúde-doença, eles têm merecido pouco respaldo no corpo da "epidemiologia clássica", ficando classificados no grupo, pouco expressivo, dos "estudos ecológicos" ou "de agregados". Tais estudos têm sido, usualmente, definidos como "empirical investigation involving the group as unit of analysis". ${ }^{49}$ Diferenciam-se dos demais desenhos de pesquisas epidemiológicas por utilizar o grupo (dados agregados), em lugar do dado individual, como unidade de análise. Apesar do caráter coletivo ou populacional do objeto da epidemiologia, os estudos que utilizam a unidade individual prevalecem sobre aqueles baseados em dados agregados, pois estes são considerados de importância menor, em termos da capacidade de demonstrar associações de causa-efeito. Vejamos as razões apresentadas pela epidemiologia "clássica".

Os estudos ecológicos sāo classificados em quatro subgrupos: a) "exploratórios", de caráter descritivo; b) “comparaçāo de múltiplos grupos”, quando ocorre análise, utilizando-se diversos grupos (p. ex., unidades geográficas) para verificar associações entre o nível médio da exposição(X) e a taxa de ocorrência da doença(Y); c) "de tendência temporal(ou de séries temporais)", que é o nosso interesse no momento, quando o objetivo é observar o relacionamento entre mudanças no nível médio de exposição (ou intervenção) e mudanças nas taxas de ocorrência da doença em uma única população, em unidades de tempo sucessivas; d) "mistos", quando se observa o relacionamento entre as mudanças no nível médio de

48. MACKENBACH J.P., STRONKS K., KUNST A.E., "The contribution of medical care to inequalities in health: differences between socio-economics groups in decline of mortality from conditions amenable to medical intervention", Soc. Sci.Med.; v.29, n.3, 1989, p.369-76.

49. MORGESTERN H., "Uses of ecologic analysis in epidemiologic research", AJPH, v.72, n.12, 1982, p.1336. 
exposição e as mudanças nas taxas de doenças entre vários grupos, em períodos sucessivos de tempo, ou seja, uma associação de b) e c).

O uso de estudos "ecológicos" na análise social é antigo. Eles estão diretamente vinculados aos censos e às formas sistemáticas de coletas de dados que objetivam, em última instância, desenvolver a capacidade administrativa do Estado contemporâneo. ${ }^{50}$ Os cientistas sociais têm utilizado amplamente dados coletados para agregados, sejam eles estados, municípios, províncias ou mesmo nações. Esses estudos têm demonstrado uma capacidade de apreender e retratar, em sua especificidade, quadros sanitários diferentes e complexos.

O uso de dados agregados, contudo, tem provocado um tipo particular de erro metodológico, denominado "falácia ecológica". A popularização deste termo deve-se ao clássico artigo de Robinson, "Ecological Correlations and the Behavior of Individuals". ${ }^{11}$ Nesse artigo, Robinson colocou seriamente em dúvida a produção de inferências causais sobre comportamentos individuais, a partir de dados agregados ou grupais. Ele procurou demonstrar que interpretar variáveis individuais, a partir da análise de unidades agregadas, é pressupor que as medidas estatísticas computadas para estas unidades devam ter os mesmos valores encontrados no nível individual.

Como exemplo paradigmático desse erro, temos o clássico trabalho de Durkheim, $O$ suicídio, ${ }^{52} \mathrm{em}$ que o autor faz uma detalhada análise deste fenômeno na Europa, utilizando em seu componente empírico dados coletados sobre a frequência de suicídios e as crenças religiosas das populações de diversas províncias contíguas da Europa Ocidental. O autor observou que, em média, províncias com maior proporção de protestantes tinham maiores taxas de suicídio, e o inverso ocorria com as províncias com maior proporção de católicos. Tais achados levaram Durkheim a teorizar sobre o papel da prática religiosa protestante como fator estimulante ao suicídio. Décadas mais tarde, novas análises dos mesmos dados permitiram verificar que a maior taxa de suicídio, em realidade, ocorria entre católicos que habitavam em áreas com predomínio de protestantes..$^{53}$ A associação encontrada, resultante da comparação entre os grupos, mos-

50. SHAW M. \& MILES I., "The social roots of statistical knowledge", in IRVINE J., MILES I e EVANS J. (ed.), Demystifying social statistics. 2a.ed., London, Pluto Press, 1981, p.27-38.

51. ROBINSON W.S., "Ecological correlations and the behavior of individuals". Am.Sociol.Rev., n.15, 1950, p.351-7.

52. DURKHEIM E., Suicide: a study in sociology. New York, The Free Press,1951.

53. SELVIN H.C., "Durkheim's "Suicide" and problems of empirical research", Am.J.Sociol., n.63, 1958, p.607-19. 
trou-se falsa, ao se tentar explicar o que ocorria no interior dos grupos. No entanto, tem-se observado que nem sempre ocorre tal grau de discrepância.$^{54}$ Investigaçōes epidemiológicas têm mostrado que, em muitos casos, é possivel obter estimativas de risco relativo em estudos com dados agregados, que se assemelham àqueles obtidos a partir de estudos longitudinais ou de caso-controle, portanto, de base individual. ${ }^{\text {ss }}$

A "falácia ecológica” tem sido a crítica mais contumaz para achados envolvendo associações causais em qualquer trabalho que utilize dados agregados. Isso explica, em grande parte, a razão pela qual a epidemiologia tem dado pouco respaldo a tais estudos.

A preocupação de Robinson sobre o uso de dados agregados tornou-se para muitos cientistas sociais, principalmente os epidemiologistas, um verdadeiro tabu. É importante observar, contudo, que também é possível concluir, a partir do estudo de Robinson, que: a) a análise de dados agregados pode ter interesse em si mesmo; b) a comparação de diferentes níveis de agregaçāo e de dados individuais pode trazer resultados interessantes. ${ }^{56}$

\section{Conclusões}

A delimitação do objeto de estudo de qualquer ciência é processual. O objeto científico se modifica no decorrer do tempo, de acordo com os desafios enfrentados em diferentes contextos.

Os estudos sobre as mudanças nos padrões de morbi-mortalidade têm passado de forma tangencial pelo núcleo central de interesses da epidemiologia "clássica". O desenvolvimento da "teoria da transição epidemiológica" foi motivado por demógrafos interessados nas implicaçôes que os padrões de transformação do fenômeno saúde-doença tiveram sobre a dinâmica populacional em diversas nações. Por outro lado, os estudos empíricos voltados para o entendimento das determinações das mudanças nos padrōes de morbi-mortalidade em uma sociedade, por causa de problemas de ordem supostamente metodológicas, são relegados a segundo plano. Pode-se dizer que a epidemiologia "clássica" tem uma forte tendên-

54. MOORMAN J.E., "Aggregation bias: an empirical demonstration", in: BORGATTA E.F. \& JACKSON D.J. (ed.), Aggregate data: analysis and interpretation, London, Sage, 1980. p:131.56.

55. RICHARDSON S., STUCKER I. \& HEMON D., "Coparison of relative risks obtained in ecological and individual studies: some methodological considerations", Int. J.Epidemiolol., v.16, n.1, 1987, p.111-20; BERAL V., CHILVERS C. \& FRASER P., "On the estimation of relative risk from statistical data", J.Epidemiol.Comm.Hlth., n.33, 1979, p.159-62.

56. BORGATTA E.F. \& JACKSON D.J., "Aggregate data analysis. An overview", in: BORGATTA E.F. \& JACKSON D.J. (ed.), Aggregate data: analysis and interpretation, op. cit., p.7-12. 
cia a minimizar sua contribuição potencial para o esclarecimento dessas mudanças.

É importante observar que as transformações populacionais geram diferentes pressões sobre os serviços de saúde. Para que a epidemiologia possa contribuir mais efetivamente para o esclarecimento desse fato é necessário que ela desenvolva procedimentos diagnósticos e terapêuticos de base populacional - social - mais ampla. Os estudos epidemiológicos podem lançar nova luz no entendimento dos determinantes da morbidade e da mortalidade de uma dada população. A apreensão dessa realidade social pela epidemiologia, contudo, exige um contínuo questionamento das bases teóricas desta disciplina.

Uma questão que tem sido insuficientemente trabalhada na epidemiologia - as relações entre o individual e o coletivo - é um desafio a enfrentar. ${ }^{57}$ Rose, ${ }^{58}$ de forma contundente, expõe os limites da epidemiologia, ao entender que a disciplina tem se concentrado nas determinações dos casos individuais e não na determinação das taxas de ocorrência dos eventos mórbidos na população. Os fatores que determinam a ocorrência de uma doença não são similares àqueles relacionados com as taxas da mesma doença. Há uma tendência, como diz Possas, de localizar os estudos epidemiológicos em "patologias específicas” que, “embora da maior relevância para o avanço do conhecimento dos mecanismos de propagação de doenças tomadas isoladamente, tem dificultado o desenvolvimento de instrumentos de análise adequados à apreensão global da realidade epidemiológica de populações concretas". ${ }^{59}$

As estratégias de investigação epidemiológica nos estudos dos determinantes da doença são insuficientes para o entendimento de como ocorrem, ou se alteram, os fenômenos coletivos. Os problemas metodológicos existentes, gerados pelos bias de agregação não existirão, quando o objetivo for obter inferências sobre os fenômenos grupais, como as taxas de mortalidade, e não sobre os casos que compõem o grupo. ${ }^{60}$ Isto não implica a inexistência de problemas e limitações metodológicas de outras ordens, inclusive aqueles específicos da análise de série temporais. ${ }^{61}$ Porém, a

57. BARRETO M.L. \& ALVES P.C., O coletivo versus o individual na epidemiologia: contradição ou sintese? Trabalho apresentado no II Congresso Brasileiro de Epidemiologia, Belo Horizonte, 1992.

58. ROSE G., "Sick individuals and sick populations", Int. J. Epidemiol., v.14, n.1, 1985,32-8.

59. POSSAS C., Epidemiologia e sociedade: heterogeneidade estrutural e saúde no Brasil. Sāo Paulo, HUCITEC, 1989. p.213

60. LANGBEIN L.I. \& LICHTMAN A.J., Ecological inference. London, Sage, 1978.

61. OSTROM C.W., Time series analysis: regression techniques. London,Sage,1978; GREENLAND S \& MORGENSTERN H, "Ecological bias, confounding, and effect modification". Int. J. Epidemiol., v.18, n.1, 1989, p.269-73. 
rejeição pura e simples dessa forma de enfocar o olhar epidemiológico implica invalidar toda a riqueza dos argumentos que têm ajudado a entender as conseqüências das transformações históricas da sociedade humana no processo saúde-doença.

\section{RESUMO}

Mudanças dos padrões de morbi-mortalidade: uma revisão crítica das abordagens epidemiológicas

O artigo analisa duas abordagens sobre as modificações dos padrões epidemiológicos ocorridas em países desenvolvidos e subdesenvolvidos: a teoria da transição epidemiológica e os estudos empíricos sobre os possíveis determinantes dessas tendências. Apesar da importância destas diferentes abordagens, a epidemiologia "clássica" tem minimizado a sua capacidade de contribuir para o esclarecimento das modificações dos padrões de morbi-mortalidade.

\section{ABSTRACT \\ Changes in the patterns of morbidity and mortality: a critical review of epidemiological approaches}

The paper aims at analysing two approaches to the changes on epidemiological patterns in developed and underdeveloped countries: the theory of epidemiological transition and the empirical studies about the possible determinants of these changes. Despite the importance of these approaches, the contribution of "classical" epidemiology to the explanation of the changes in patterns of morbidity and mortality has been of small significance. 


\section{RESUME}

Les changements des modèles de morbi-mortalité: une révision critique des abordages epidémiologiques

L'article analyse deux abordages sur les modifications des modèles épidémiologiques survenus dans les pays développés et sous-développés: la théorie de transition épidémiologique et les études empiriques sur les facteurs probables de ces tendances. En dépit de l'importance de ces différents abordages, l'épidemiologie "classique" a minimisé ses capacités de contribution pour l'explication des modifications des modèles de morbi-mortalité. 